\title{
Efficacy and safety of lumen-apposing metal stent for benign gastrointestinal stricture
}

\author{
Deepanshu Jain ${ }^{a}$, Upen Patel ${ }^{b}$, Sara Ali ${ }^{b}$, Abhinav Sharma ${ }^{b}$, Manan Shah ${ }^{b}$, Shashideep Singhal ${ }^{b}$
}

Einstein Healthcare Network, Philadelphia, PA; University of Texas Health Science Center at Houston, Houston, Texas, USA

\section{Abstract}

\begin{abstract}
Management of benign gastrointestinal (GI) strictures refractory to primary (balloon and savary dilation) and secondary (steroid injection, fully covered self-expanding metal stent, incision therapy) treatment modalities remains a challenge. Lumen-apposing metal stents (LAMSs), originally designed for the management of pancreatic fluid collections, are an attractive option for GI stricture because of their anti-migratory property, attributable to their saddle-shaped design. In this article, we reviewed 70 patients from 12 original studies who received LAMS for refractory (68/70) or treatment-naive (2/70) benign GI stricture. The technical and clinical success rates were 98.6\% (69/70) and 79.7\% (55/69), respectively. Endoscopic placement, with or without fluoroscopic guidance, was generally successful, with only a minority requiring endoscopic ultrasound (EUS) guidance where the lumen was completely obscured. The majority of the strictures were short $(\leq 1 \mathrm{~cm})$, but comparable technical and clinical success was noted in isolated cases with long strictures, where 2 overlapping LAMSs were placed. For the overall population, a failure rate of $21.5 \%(14 / 69)$ was noted and was attributed to either lack of follow up, or to persistent or de novo symptoms requiring stent removal/exchange or surgical referral. One perforation (1.4\%), five stent migration events (7.1\%), two bleeding events (2.9\%) and two de novo strictures proximal to the LAMS (2.9\%) were reported for the entire study cohort. No mortality was attributable to LAMS placement. Although experience is still evolving, LAMS placement guided by esophagogastroduodenoscopy or EUS is a technically feasible and safe procedure with good clinical outcomes for benign refractory GI strictures.
\end{abstract}

Keywords Benign stricture, gastrointestinal stricture, lumen-apposing metal stent

Ann Gastroenterol 2018; 31 (4): 1-14

\section{Introduction}

Benign gastrointestinal (GI) stricture can arise in any part of the GI tract as a result of various etiologies. However, it usually occurs in the esophagus and pyloric channel. Anastomotic sites are also potential locations for benign GI strictures [1]. Endoscopic dilation, incisional therapy, intralesional steroid

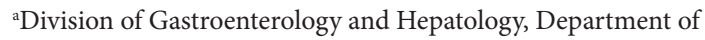
Digestive Diseases and Transplantation, Internal Medicine, Einstein Healthcare Network, Philadelphia, PA (Deepanshu Jain); ${ }^{\text {DDivision }}$ of Gastroenterology, Hepatology and Nutrition, University of Texas Health Science Center at Houston, Houston, Texas (Upen Patel, Sara Ali, Abhinav Sharma, Manan Shah, Shashideep Singhal), USA

Conflict of Interest: None

Correspondence to: Shashideep Singhal, MD, Division of Gastroenterology, Hepatology and Nutrition, University of Texas Health Science Center at Houston, 6431 Fannin, MSB 4.234, Houston, Texas, USA 77030, e-mail: sdsinghal@gmail.com

Received 8 January 2018; accepted 24 February 2018; published online 7 May 2018

DOI: https://doi.org/10.20524/aog.2018.0272 injection and self-expandable metal stents (SEMSs) are the available treatment modalities for GI stricture [2-4]. Although these treatments are safe and effective, there are certain drawbacks: endoscopic dilatation is associated with high recurrence, requiring multiple treatment sessions [5], while SEMSs are vulnerable to stent migration [6]. SEMSs are estimated to have a $30-40 \%$ migration rate or greater $[7,8]$, which could be minimized by fastening with over-the-scope clips [9] and endoscopic suturing. [10]. However, stent migration rates still exceed $15 \%$ [1].

The lumen-apposing metal stent (LAMS) (Axios stent, Boston Scientific, Marlborough, Mass.) is a saddle-shaped stent (10 $\mathrm{mm}$ in length, $10 \mathrm{~mm}$ and $15 \mathrm{~mm}$ in diameters, with wide flanges of $23 \mathrm{~mm}$ and $28 \mathrm{~mm}$ ), which achieves lumen apposition because of its bilateral anchoring flanges, thus decreasing the risk of stent migration [11]. LAMSs were initially used to drain pancreatic fluid collections [12]; however, they have increasingly been utilized in the management of benign GI stricture because of their anti-migratory lumen-apposing design.

In this review article, we have summarized case reports, case series and retrospective studies to evaluate the safety, efficacy and feasibility of LAMS in the management of benign GI stricture. 


\section{Materials and Methods}

An extensive search of the English language literature until July 2017 was performed, using PubMed and Google Scholar to identify the peer-reviewed original and review articles based on the key words "benign stricture", "gastrointestinal stricture" and "lumen-apposing metal stent". Only articles that concerned human study subjects were selected. The reference lists of relevant studies were manually searched to identify additional further appropriate publications. The search yielded 9 case reports [13-21], 1 case series [22], and 2 retrospective studies [1,11].The stricture etiology, characteristics, location, device details, procedure details, success rate, complications, and limitations in each study were reviewed.

\section{Results}

Twelve original articles were included in this review [1,11,13-22]. Two retrospective multicenter studies from the USA $[1,11] ; 9$ case reports, 7 from the USA [13$16,18,20,21]$ and 2 from Spain [17,19]; and 1 case series from the USA [22] were included in the review. In this article, we reviewed 70 patients from the 12 original studies who received LAMS for refractory (68/70) or treatment-naïve (2/70) benign GI strictures. The technical and clinical success rates were $98.6 \%(69 / 70)$ and $79.7 \%$ (55/69), respectively. Endoscopic placement, with or without fluoroscopic guidance, was generally successful, with only a minority requiring endoscopic ultrasound (EUS) guidance where the lumen was completely obscured. The majority of strictures were short $(\leq 1 \mathrm{~cm})$, but comparable technical and clinical success was noted in isolated cases with long strictures, where 2 overlapping LAMSs were placed. The overall study population had a failure rate of $21.5 \%$ (14/69), attributed to either lack of follow up, or to persistent or de novo symptoms requiring stent removal/exchange or surgical referral. One perforation (1.4\%), five stent migration events $(7.1 \%)$, two bleeding events (2.9\%) and two de novo strictures proximal to the LAMS (2.9\%) were reported for the entire study cohort. No mortality was attributable to LAMS placement. The results from all the studies are summarized in Table 1.

\section{Discussion}

\section{Patient characteristics}

GI strictures usually present with symptoms of dysphagia, abdominal pain, cramping, weight loss or other symptoms of obstruction. The age of the patients ranged from 18-86 years across the included studies $[1,11,13-22]$ and the majority (67.1\%) were female [1,11,13-22].

\section{Stricture characteristics}

\section{Etiology and location}

In this review, all 12 studies included patients with benign GI stricture [1,11,13-22]. These strictures were distributed along the entire GI tract. Of the 70 strictures, 8 were at an esophagogastric anastomotic site $[1,11], 32$ were at a gastrojejunal anastomotic site $[1,11,13,14,18,21,22], 13$ were at the pylorus $[1,11,16,22], 7$ were at a colon anastomotic site $[11,22], 2$ were at a rectal anastomotic site [19,20], while 1 stricture was located at each of the following locations: ileorectal anastomotic site [1], gastric [1], esophageal anastomotic site [14], colorectal anastomotic site (Crohn's disease) [15], esophagogastric junction [11], colon [11], rectosigmoid anastomotic site [20], and gastrojejunostomy site [22]. The majority of the strictures were post-surgical anastomotic strictures [1,13-15,17-22], but there were also strictures secondary to long-standing inflammation, such as peptic ulcer disease $[11,16,18,22]$. Surgical interventions, including esophagectomy, Roux-en-Y bypass, gastroplasty, Billroth II and colectomy, performed to treat primary malignancy, inflammatory bowel disease or obesity, were the most common underlying etiology [1,13-15,17-22].

\section{Length, diameter, and chronicity}

Only a few authors reported the length and/or diameter of the stricture and this varied widely across different studies and within each study $[1,11,14,15,19,21,22]$. The longest stricture was reported to be $4 \mathrm{~cm}$ in length at a gastrojejunal anastomosis [22]. In this particular case, the authors described the use of two overlapping LAMSs to allow complete bridging of the stricture [22]. The majority of the other strictures were $\leq 1 \mathrm{~cm}$ in length $[1,11,14,15]$. The luminal diameter of all strictures with available data was reported as less than $10 \mathrm{~mm}[11,14,19,21,22]$.

Only two studies reported the duration of the stricture [1,15], which generally ranged from 81 to 204 days [1], though in one case the stricture was reported to be there for more than 3 years [15].

\section{Prior interventions}

Although 3 studies reported no use of prior alternative treatment modalities $[16,17,20]$, in the majority of the cases reviewed the strictures were long-standing and various other treatment modalities had been attempted before LAMS was utilized $[1,11,13-15,18,19,21,22]$. One study mentioned the use of steroid injections [1], one study used a topical mesalamine and steroid enema [15], 1 study reported the needle knife technique for esophagogastric anastomotic strictures [1], 4 studies described conventional fully covered self-expanding metal stents $[1,11,14,22]$, and 9 studies mentioned endoscopic dilation $[1,11,13-15,18,19,21,22]$ as a first-line therapy in the management of various benign GI strictures. Repeated dilations of a persistent/recurrent stricture pose a higher risk of 


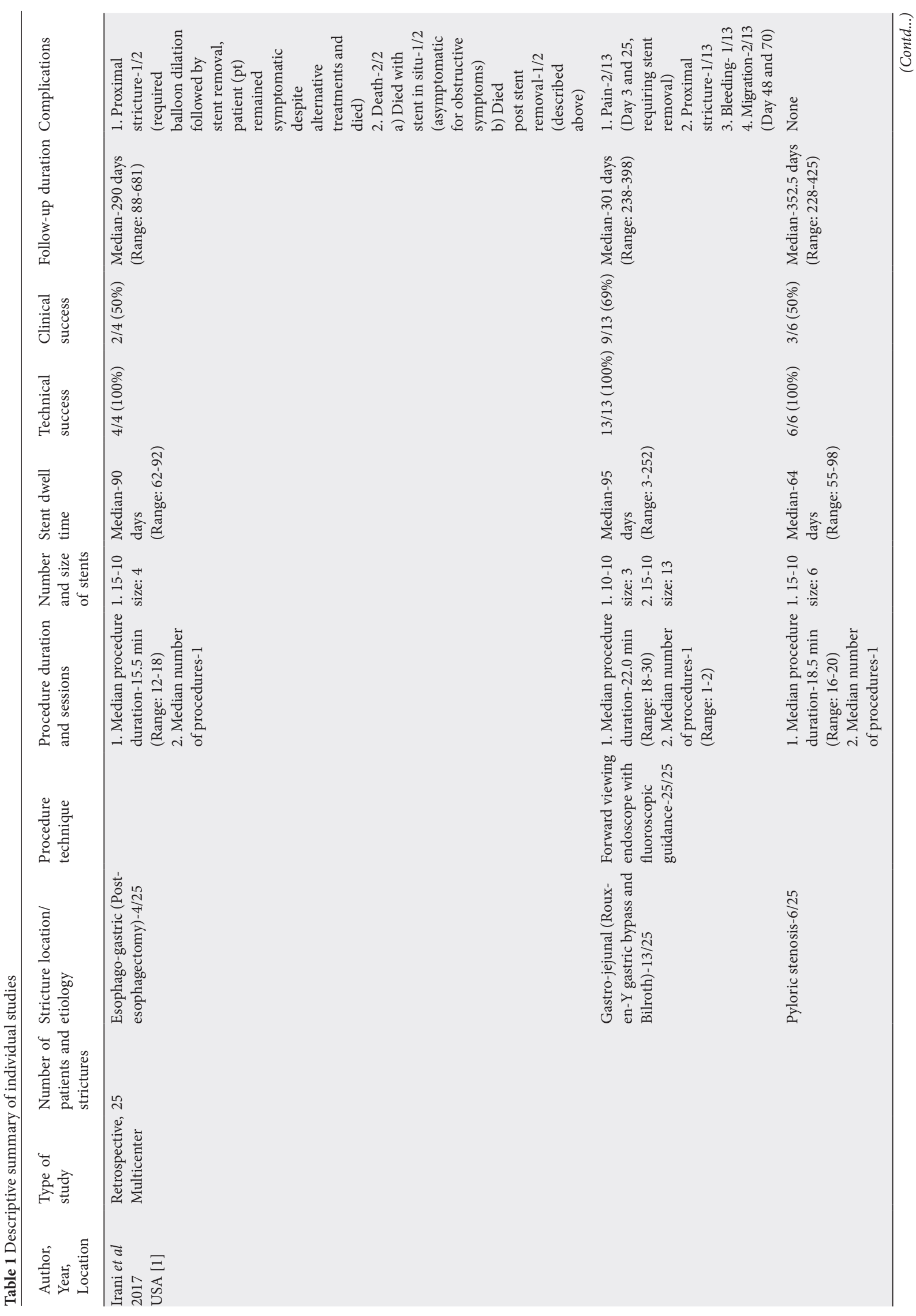


4 D. Jain et al.

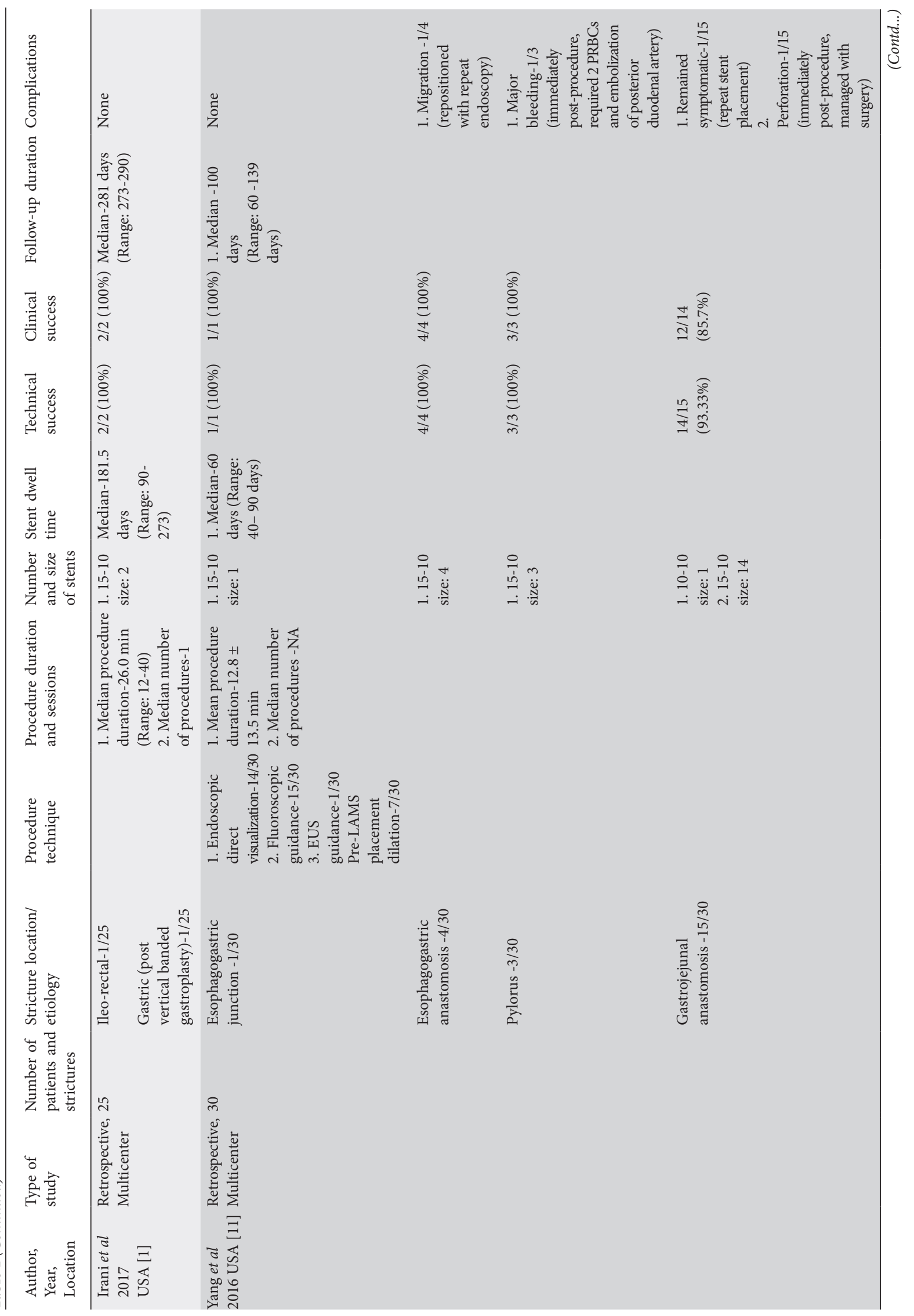




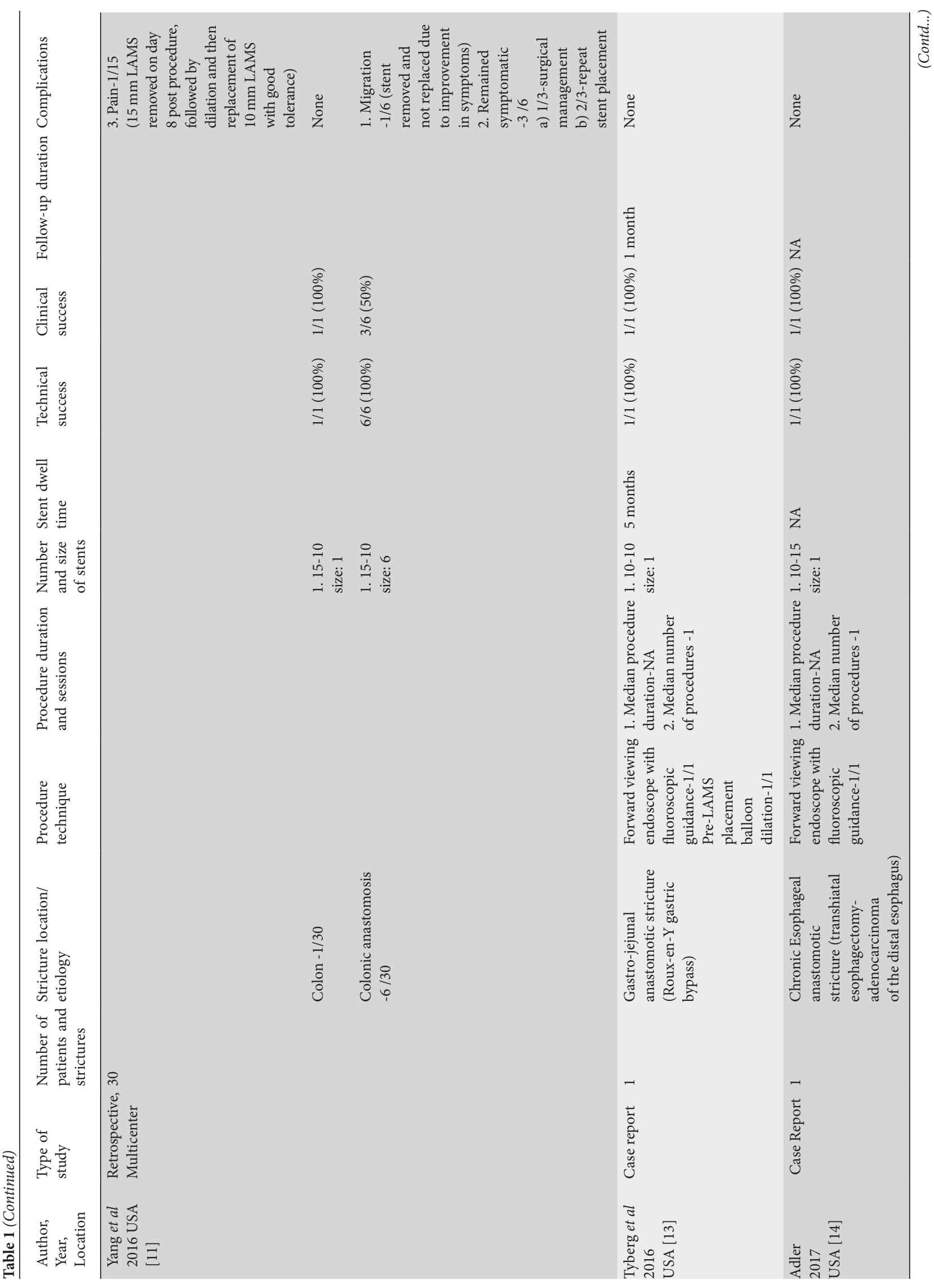




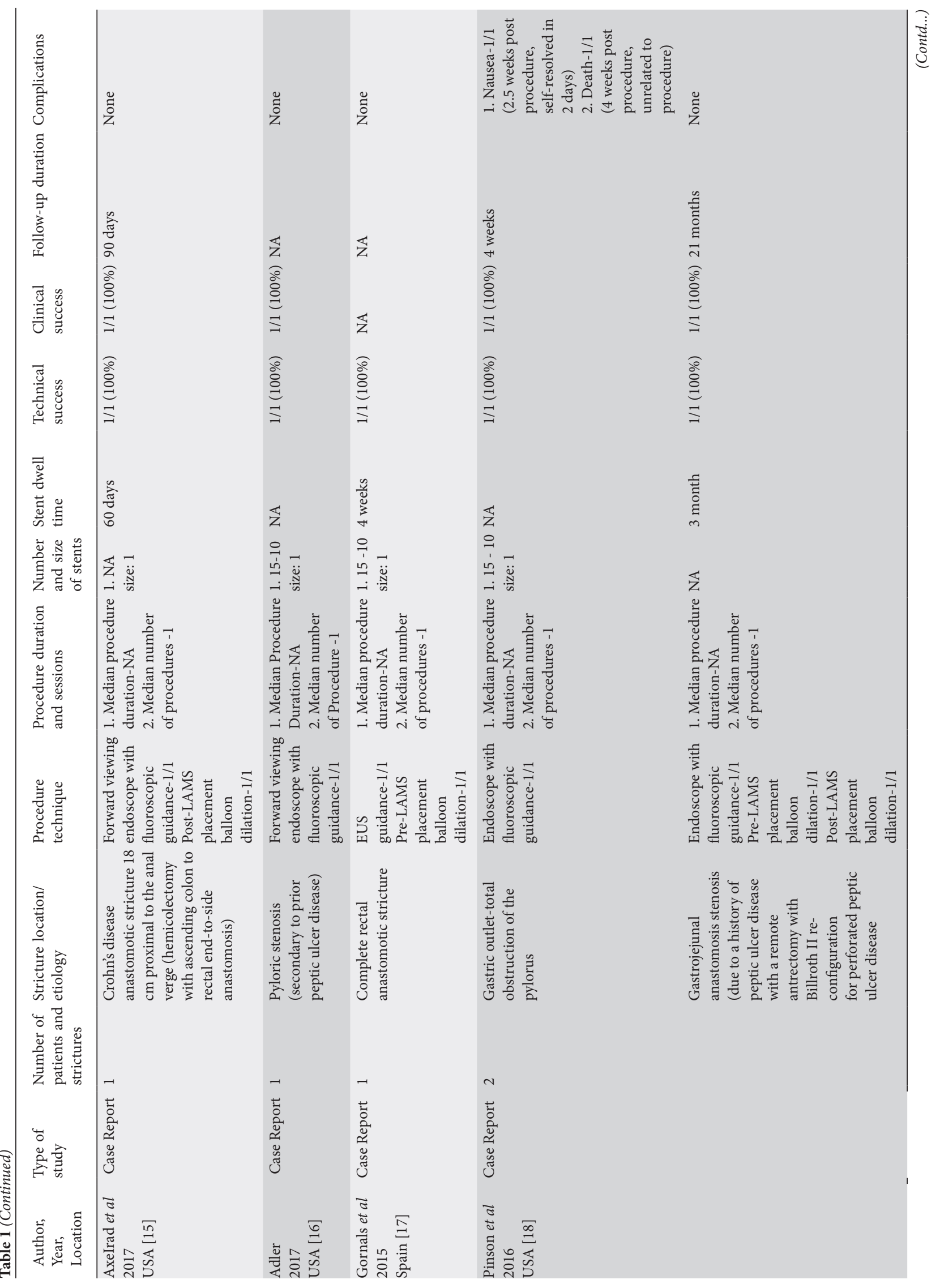




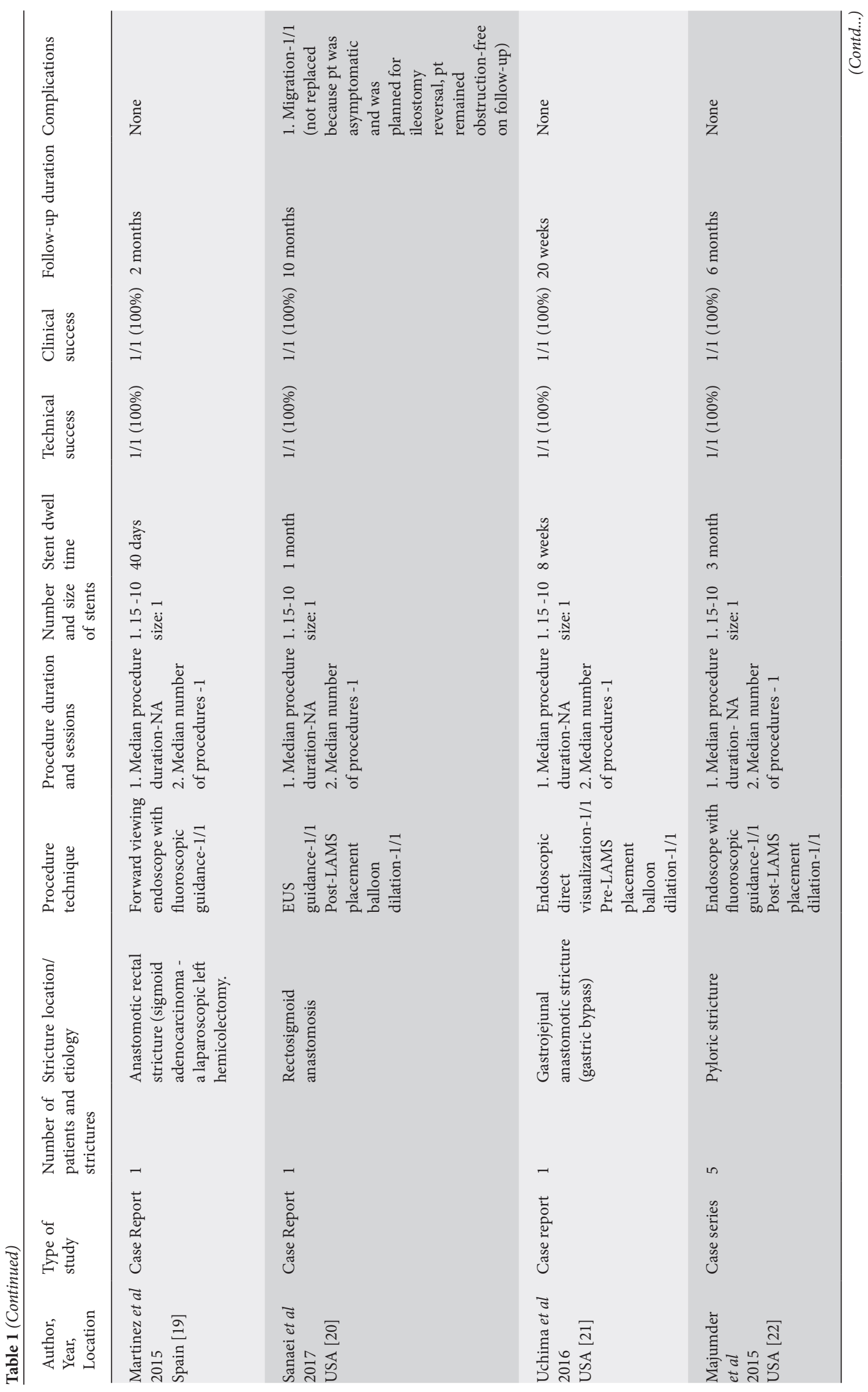


8 D. Jain et al.

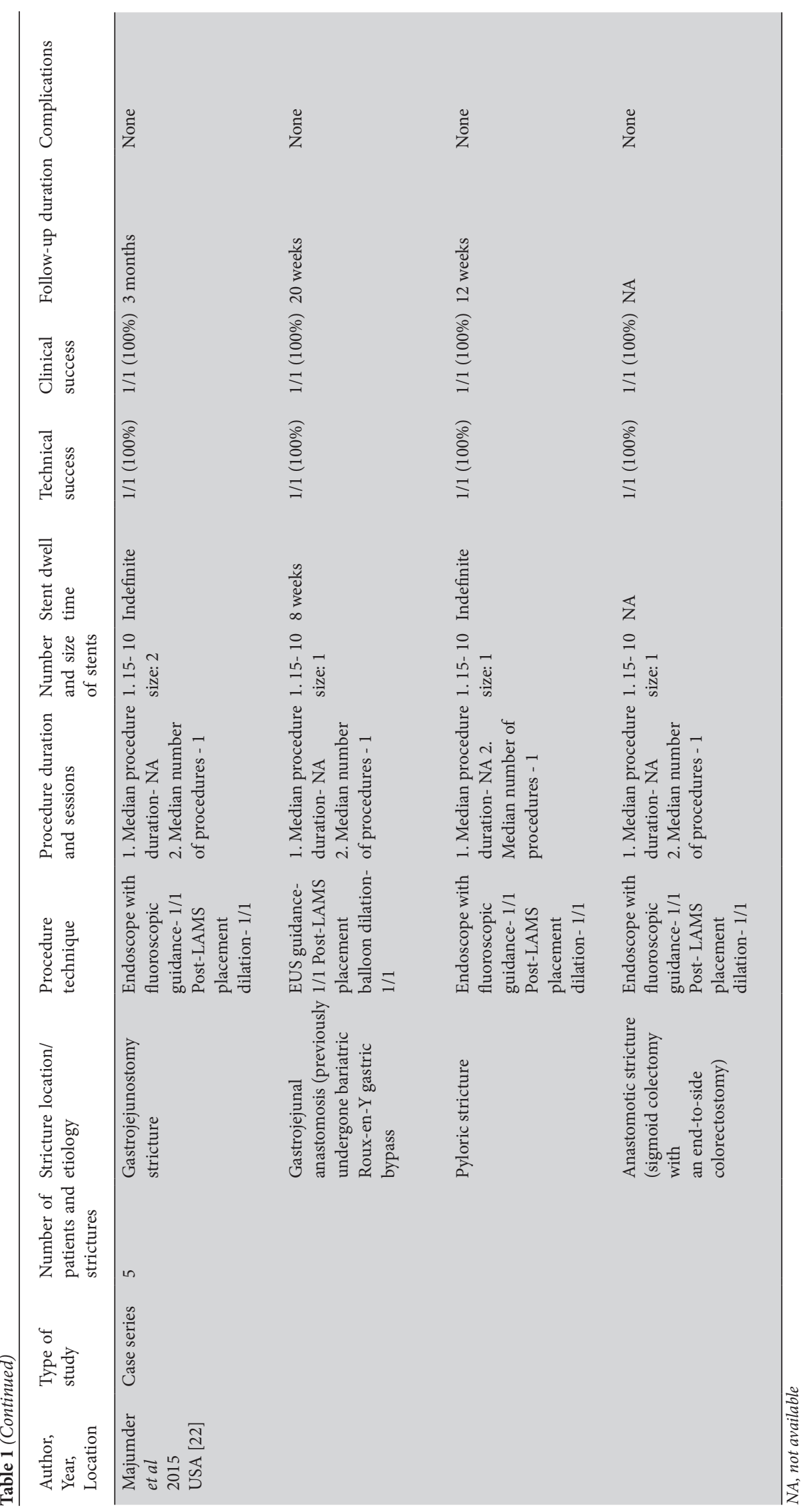


bowel perforation with each attempt and a LAMS may be a safer and efficacious alternative. Patient and stricture characteristics from each study are summarized in Table 2.

\section{Procedure characteristics}

\section{Technique}

The LAMS is a novel saddle-shaped stent. It is $1 \mathrm{~cm}$ long, 10 or $15 \mathrm{~mm}$ in diameter and has wide flanges of $23 \mathrm{~mm}$ and $28 \mathrm{~mm}$ diameter on its ends. Placement of a LAMS across a stricture requires either direct visualization via endoscopy, with $[1,11,13-16,18,19,22]$ or without [11,21] fluoroscopic assistance, or an EUS-guided approach [11,17,20,22]. If the diameter of the stricture lumen is too narrow or obstructed to allow safe passage of a guide-wire, authors have described using EUS to confirm the lumen across the stricture. In such a scenario, the lumen on the other side is preferentially filled with fluid to allow distention and visualization by EUS. This is followed by insertion of needle a across the stricture, followed by the guide-wire and LAMS placement. For both the endoscopic and the EUS-guided approach, a few studies report preferentially dilating the stricture with a balloon to allow LAMS placement $[11,13,17,18,21]$. In addition, a minority of studies also report balloon dilation post-LAMS placement to distend the LAMS to its maximum diameter [15,18,20,22].

In our review of 70 patients, $72.9 \%$ (51/70) underwent fluoroscopy-guided endoscopy, 21.4\% (15/70) direct visualization with endoscopy and 5.7\% (4/70) had EUS-guided placement of the LAMS across the stricture site. Pre-LAMS balloon dilation was performed in 10 patients $[11,13,17,21]$, post-LAMS dilation in $7[15,20,22]$, while one patient had both pre- and post-LAMS balloon dilation [18]. Fig. 1 depicts the placement of a LAMS across a gastrojejunal anastomotic stricture.

\section{Duration}

Only two studies mentioned the procedure duration for LAMS placement. The median procedure duration across the studies was $19.5 \mathrm{~min}$ ranging from 15.5-26 $\mathrm{min}[1,11]$.

\section{Stent in situ duration}

Stent dwell time depended on the etiology and indication for the LAMS placement and accordingly varied from 4 weeks to an indefinite time $[1,11,13-22]$. LAMS was used as the primary treatment modality, a bridge to surgery, or as a palliative measure. Procedure and stent details from each individual study are summarized in Table 1.

\section{Outcome}

\section{Technical success and failure}

Technical success was defined as the ability to place a LAMS across the stricture. Of the 70 patients treated for stricture, technical success was achieved in 69 (98.6\%) [1,11,13-22]. In the single failure, perforation requiring surgical management was reported. [11]. The majority of studies reported a $100 \%$ success rate on an individual basis $[1,13-22]$. There was no difference in technical success for strictures of different etiology or at different locations along the entire GI tract [1,11,13-22]. In addition, strictures of all lengths, diameters, chronicity and with prior failed interventions had similar technical success rates across all the studies [1,11,13-22].

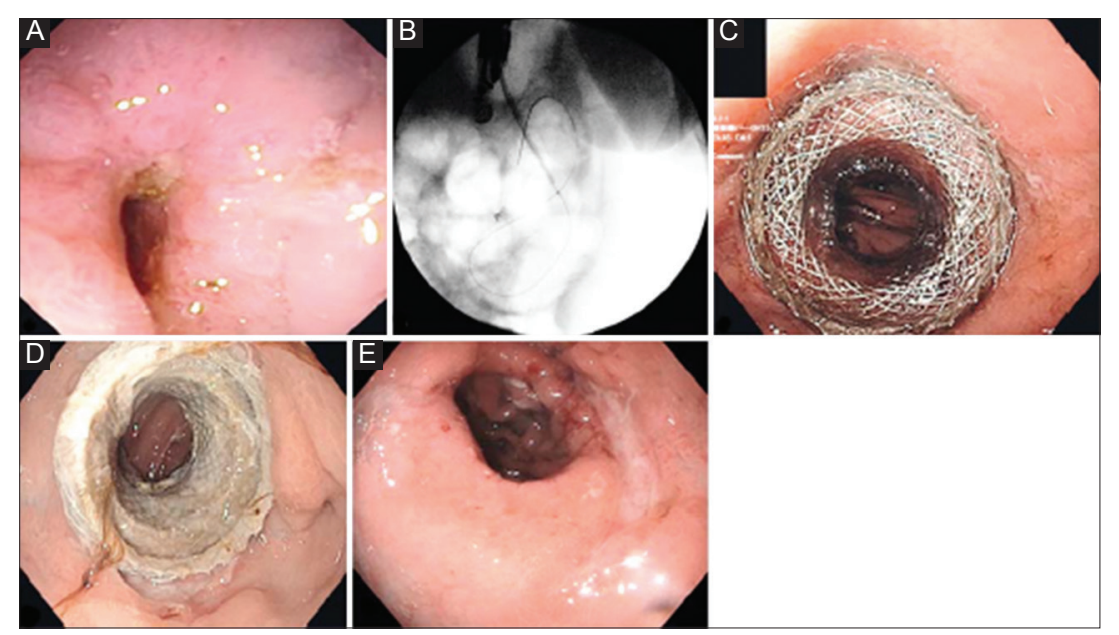

Figure 1 Placement of a lumen-apposing metal stent (LAMS) for benign gastrointestinal stricture. (A) Gastrojejunal anastomotic stricture. (B) Insertion of the LAMS over the guide-wire under fluoroscopy. (C) Endoscopic view of the successfully placed LAMS. (D) Follow-up endoscopy (54 days after insertion) showing patent LAMS across gastrojejunal anastomosis. (E) Long-term follow up (45 days after stent removal) confirming patent gastrojejunal anastomosis 
10 D. Jain et al.

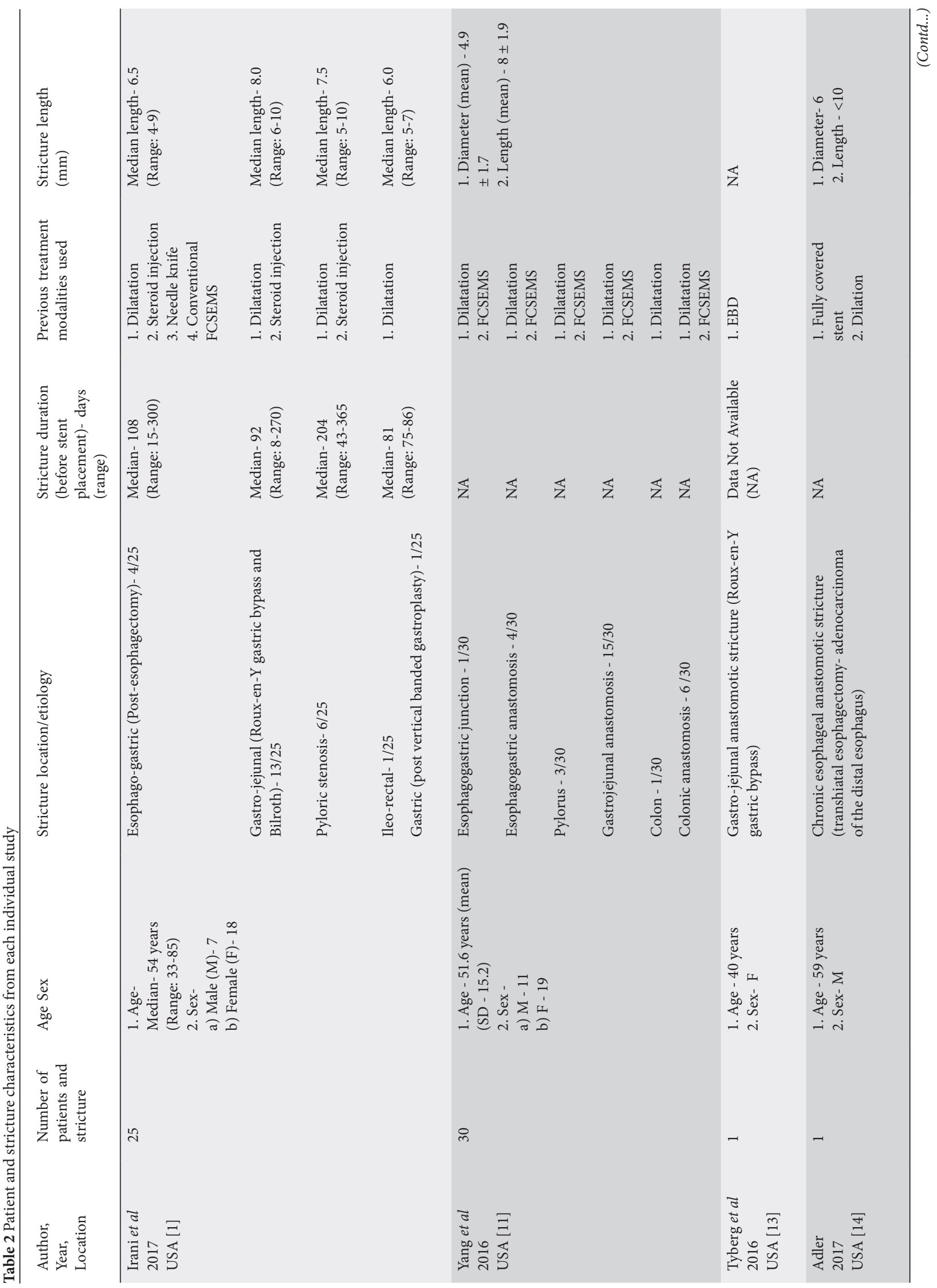




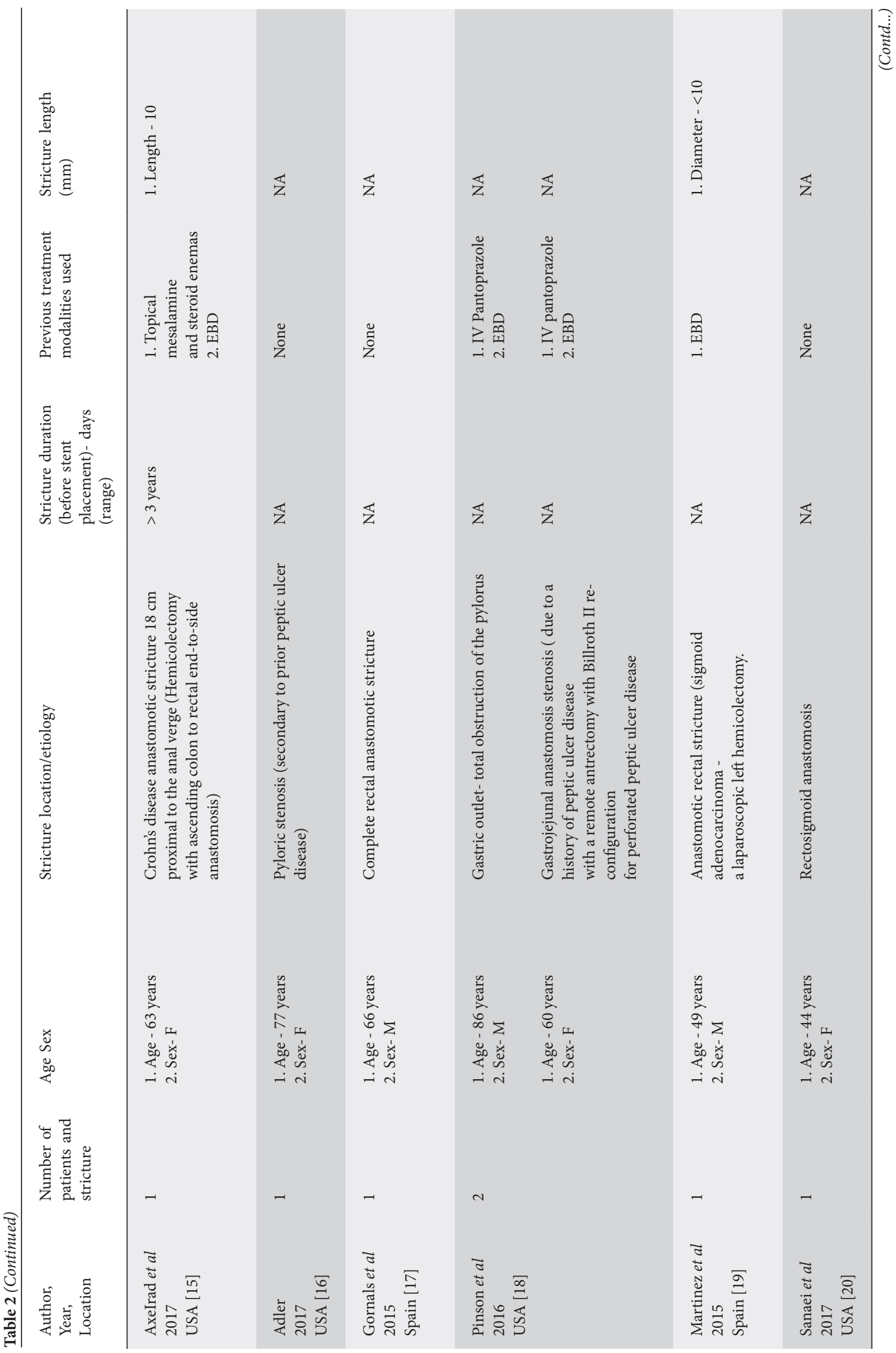


12 D. Jain et al.

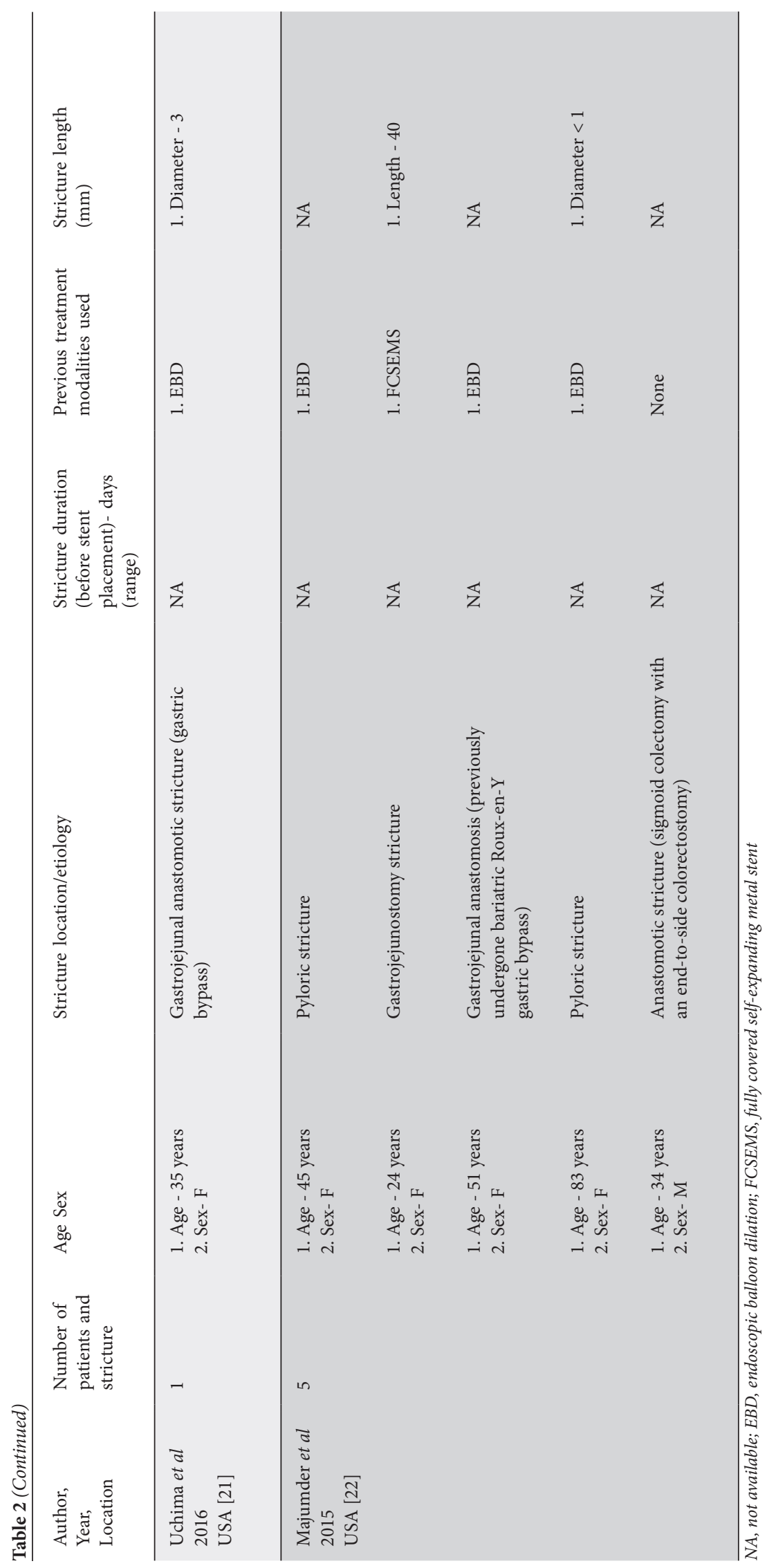




\section{Clinical success and failure}

Clinical success was described in term of the alleviation of GI obstructive symptoms, such as nausea, vomiting, constipation, abdominal pain and abdominal distension. Follow-up duration varied from as short as 30 days to as long as 21 months [1,11,1322]. The composite clinical success rate for the study cohort was $79.7 \%$ (55/69). The majority of individual studies had a $100 \%$ success rate over the study-specific follow up [13-22]. Irani et al and Yang et al reported clinical success rates of 64\% (16/25) and $82.8 \%(24 / 29)$, respectively $[1,11]$. The majority of patients $(94.2 \%$, $65 / 69)$ had some form of prior treatment for their stricture. The clinical success rate for treatment-naïve patients was $100 \%(4 / 4)$, while for refractory strictures it was $78.5 \%(51 / 65)$. The failures (14/65-21.5\%) were attributed either to lack of follow up at the study-specific time interval because of death (1/14) [1], or to the persistence of symptoms (13/14) [1,11] requiring either early stent removal, replacement or surgery referral.

\section{Complications}

On an individual basis, 8 studies reported no complications [13-17,19,21,22]. The composite complication rate could not be calculated for the study cohort because multiple adverse events were recorded per patient, which would lead to an erroneously high complication rate. To avoid this systemic error, we calculated individual event rates for the whole study cohort.

\section{Perforation}

One patient $(1.4 \%)$ developed perforation at the time of LAMS placement $[1,11,13-22]$, categorized by the study as technical failure [11]. The patient was successfully managed with surgery.

\section{Abdominal pain}

Four patients (5.7\%) had persistent symptoms [1,11,13-22]; $75 \%$ (3/4) were managed with repeat stent placement and $25 \%$ $(1 / 4)$ were referred for surgical treatment [11]. Three patients (4.3\%) developed new-onset abdominal pain on day 3 , day 8 and day 25 post-LAMS placement, requiring stent removal $[1,11]$. One of the three patients initially had a $15 \times 10 \mathrm{~mm}$ stent, replaced with $10 \times 10 \mathrm{~mm}$ stent, resulting in good tolerance [11].

\section{Stent migration}

Five patients (7.1\%) exhibited stent migration [1,11,13-22]. Of these, two were asymptomatic (requiring no reposition or replacement) [11,20], one patient underwent endoscopic repositioning [11], and for the other two no specific management was reported [1].

\section{Bleeding}

Two patients (2.9\%) had bleeding post-LAMS placement $[1,11,13-22]$. One patient had a significant bleed requiring embolization of the posterior duodenal artery [11]. The other bleeding event was associated with stent migration [1].

\section{De-novo proximal stricture}

Two patients $(2.9 \%)$ were reported to have developed a new stricture at the proximal end of the LAMS [1,11,13-22]. One of these patients required balloon dilation of the proximal stricture to allow LAMS removal [1].

\section{Mortality}

Three patients (4.3\%) died during follow up [1,11,1322 ]. None of the deaths were secondary to LAMS placement and all were attributed to these patients' underlying comorbidities $[1,18]$.

\section{Miscellaneous}

One patient (1.4\%) was reported to have developed nausea approximately 2.5 weeks post-LAMS placement [1,11,13-22] and was successfully treated with conservative management. The stent was found to be in place and patent [18].

\section{Concluding remarks}

Benign GI strictures of ischemic and inflammatory etiology can develop anywhere in the GI tract, depending on the site of previous surgery (anastomosis) or as a result of peptic ulcer disease. Endoscopic balloon dilation and savary dilation represent the primary go-to treatment modalities. Intra-lesion steroid injection, fully covered self-expandable metal stents, biodegradable stents and endoscopic incision therapy are available options for the treatment of refractory strictures, with a variable response. LAMSs, originally designed for pancreatic fluid collection drainage, appear to be a beneficial option for the management of refractory GI strictures because of their saddle-shaped design. In this article, we reviewed 70 patients from 12 original studies who received LAMS for refractory $(68 / 70)$ and treatment-naïve (2/70) benign GI strictures. Technical and clinical success rates were 98.6\% (69/70) and $79.7 \%$ (55/69) respectively. An endoscopic approach, with or without fluoroscopic guidance, allowed successful placement, with only a minority of cases requiring EUS guidance where the lumen was completely obscured. The majority of strictures were short $(\leq 1 \mathrm{~cm})$ but comparable technical and clinical success were noted in isolated cases with long strictures where 2 overlapping LAMSs were placed. For the overall study population, the failure rate was $21.5 \%$ (14/69) and was attributed to either a lack of follow up, or to persistent or de 
novo symptoms requiring stent removal/exchange or surgical referral. One perforation (1.4\%), five stent migration events (7.1\%), two bleeding events (2.9\%) and two de novo strictures proximal to the LAMS (2.9\%) were reported for the entire study cohort. No mortality was attributable to LAMS placement. Esophagogastroduodenoscopy or EUS-guided placement of a LAMS is a technically feasible and safe procedure with good clinical outcomes for benign refractory GI strictures. Head-tohead comparison trials with alternative treatment modalities are needed to ascertain any superiority or inferiority of one over the other.

\section{References}

1. Irani S, Jalaj S, Ross A, Larsen M, Grimm IS, Baron TH. Use of a lumen-apposing metal stent to treat GI strictures (with videos). Gastrointest Endosc 2017;85:1285-1289.

2. Agnew SR, Pandya SP, Reynolds RP, Preiksaitis HG. Predictors for frequent esophageal dilations of benign peptic strictures. Dig Dis Sci 1996;41:931-936.

3. Ramage JI Jr, Rumalla A, Baron $\mathrm{TH}$, et al. A prospective, randomized, double-blind, placebo-controlled trial of endoscopic steroid injection therapy for recalcitrant esophageal peptic strictures. Am J Gastroenterol 2005;100:2419-2425.

4. Jain D, Sandhu N, Singhal S. Endoscopic electrocautery incision therapy for benign lower gastrointestinal tract anastomotic strictures. Ann Gastroenterol 2017;30:473-485.

5. Pereira-Lima JC, Ramires RP, Zamin I Jr, Cassal AP, Marroni CA, Mattos AA. Endoscopic dilation of benign esophageal strictures: report on 1043 procedures. Am J Gastroenterol 1999;94:1497-1501.

6. Gangloff A, Lecleire S, Di Fiore A, et al. Fully versus partially covered self-expandable metal stents in benign esophageal strictures. Dis Esophagus 2015;28:678-683.

7. Hirdes MM, Siersema PD, Vleggaar FP. A new fully covered metal stent for the treatment of benign and malignant dysphagia: a prospective follow-up study. Gastrointest Endosc 2012;75:712-718.

8. Ham YH, Kim GH. Plastic and biodegradable stents for complex and refractory benign esophageal strictures. Clin Endosc 2014;47:295-300.

9. Irani S, Baron TH, Gluck M, Gan I, Ross AS, Kozarek RA. Preventing migration of fully covered esophageal stents with an over-the-scope clip device (with videos). Gastrointest Endosc 2014;79:844-851.

10. Fujii LL, Bonin EA, Baron TH, Gostout CJ, Wong Kee Song LM. Utility of an endoscopic suturing system for prevention of covered luminal stent migration in the upper GI tract. Gastrointest Endosc 2013;78:787-793.

11. Yang D, Nieto JM, Siddiqui A, et al. Lumen-apposing covered selfexpandable metal stents for short benign gastrointestinal strictures: a multicenter study. Endoscopy 2017;49:327-333.

12. Walter D, Will U, Sanchez-Yague A, et al. A novel lumen-apposing metal stent for endoscopic ultrasound-guided drainage of pancreatic fluid collections: a prospective cohort study. Endoscopy 2015;47:63-67.

13. Tyberg A, Desai A, Zerbo S, Nieto J, Kahaleh M. Endoscopic management of an anastomotic stricture using a lumen-apposing metal stent. Gastrointest Endosc 2016;83:464-465.

14. Adler DG. Esophageal placement of a lumen-apposing metal stent in a patient with a chronic anastomotic stricture. Gastrointest Endosc 2017;85:1291-1293.

15. Axelrad J, Lichtiger S, Sethi A. Treatment of Crohn's disease anastomotic stricture with a lumen-apposing metal stent. Clin Gastroenterol Hepatol 2018;16:A25-A26.

16. Adler DG. Use of a lumen-apposing metal stent to treat pyloric stenosis. Gastrointest Endosc 2017;86:742-743.

17. Gornals JB, Albines G, Trenti L, Mast R, Frago R. EUS-guided recanalization of a complete rectal anastomotic stenosis by use of a lumen-apposing metal stent. Gastrointest Endosc 2015;82:752.

18. Pinson R, Pathirana I, Magulick J, Domanski J, Okoh E, Womeldorph C. Novel use of a uniquely designed, lumenapposing, metal stent in benign gastric outlet obstruction in two patients. ACG Case Rep J 2017;4:e20.

19. Martínez Alcalá F, Martínez-Alcalá García FR, Sánchez-Yague A, Martínez-Alcalá García A, Ciria Avila JA, Perez Pozo JM. Treatment of a benign, anastomotic refractory rectal stricture with an AXIOS stent. Endoscopy 2015;47 (Suppl 1 UCTN): E413-E414.

20. Sanaei O, Ngamruengphong S, Chen YI, et al. Endoscopic ultrasonography-guided freestyle rendezvous recanalization of a complete postoperative rectosigmoid anastomotic obstruction with a lumen-apposing metal stent. Endoscopy 2017;49:E206-E207.

21. Uchima H, Abu-Suboh M, Mata A, Cruz M, Espinos J. Lumenapposing metal stent for the treatment of refractory gastrojejunal anastomotic stricture after laparoscopic gastric bypass. Gastrointest Endosc 2016;83:251.

22. Majumder S, Buttar NS, Gostout C, et al. Lumen-apposing covered self-expanding metal stent for management of benign gastrointestinal strictures. Endosc Int Open 2016;4:E96-E101. 